\title{
Understanding the Ultimate Structure of Matters and Some Scientific Doubts of Nature through Simple Hypotheses
}

\author{
Bao-Yu Zong \\ (Temasek Laboratories, National University of Singapore, Singapore)
}

\begin{abstract}
There are numerous unanswered scientific doubts/phenomena in Nature which still perplex scientific researchers currently. To solve these puzzles, unveiling the ultimate structure of matters, which make up the Nature world, is essential. Here four hypotheses, namely the fundamental matter component (basic particle) and its property, construction process of the basic particles into other particles/matters, repulsion of particles of the same type, and direct relationship between matter and energy, are proposed. These simple hypotheses can well explain some natural phenomena, such as the existence of huge atom energy and various particles/matters of different properties, and conversion balance between matters and energy. Due to the existence of diverse infinite fine particles and repulsion of particles of the same types, matters and the Nature world are submerged in a sea of particles and there is not a real vacuum space (without any particle) in the Nature world. This in turn reveals Thermal Laws, the component of dark energy/matter, as well as a minimum temperature of $\sim 3 \mathrm{~K}$ that exists in the deep Universe. The suggested relationships between particles and/or matters also shed light on the mechanisms of interaction forces of same or different polarity particles, and intermedia of gravitational, electrical and magnetic fields.
\end{abstract}

Keywords: Basic particle, elementary particle, dark energy/matter, smallest transporter of energy, fundamental component of matter

\section{Introduction}

The ultimate structure of matters is still unclear nowadays although it is crucial for the realization of Nature laws and numerous scientific puzzles $[1,2]$. Since the discovery of the heat rays (photons) by William Herschel in 1800 [3], numerous particles (viz., the matters with a density and dimension equal to or less than a gas molecule), such as atom, electron, atomic nucleus, proton, neutron, muon, pion, kaon, antielectron (or positron), antiproton, Xi baryon, partons, top quark, tau neutrino, Higgs boson, and so on, have been consecutively found [4 - 7]. From these discoveries, the Nature and matters are known to comprise of numerous types of particles; diverse matters consist of different atoms, which contain varying number of protons, neutrons and electrons [8]. Despite of these impressive progresses on the understanding of the structure of matters, there are still numerous doubts unsolved regarding matters, such as the relation between and structures of different particles, components which make up the electrical, magnetic and gravitational field media, direct relationship between matters and energy, origin of great energy stored in atoms (e.g., atom- or hydrogen-bomb), root-causes of scattering when particle beams moving forward, as well as conversion balance between energy and matters [9]. To solve these puzzles, it is impossible if the configurations of all particles or the ultimate structure of matters are not clear since the well-known and unknown particles make-up of all the matters and Nature world. In order to exploit the ultimate structure of matters, different theories (such as the Standard Model and Higgsless Model of particle physics) have been set up [10]. According to the Standard Model, some elementary particles are defined, such as the fundamental fermions (quarks, leptons, antiquarks, and antileptons, viz, matter and antimatter particles) and bosons (gauge boson and the Higgs boson, viz, force particles, mediating interactions among fermions). However, although the Higgs boson, as the elementary particle, has been identified by means of a high speed/energy of proton collision using Large Hadron Collider (LHC) [11], there is no full confidence in these theoretical models for addressing the above mentioned scientific doubts $[9,10]$. For instance, it remains unclear what are the substructures of the elementary particles and whether other finer particles make-up of these elementary particles. In addition, it is also unknown whether the elementary particles and other particles (e.g., electrons, photons) can be decomposed or decayed to sparser/finer particles. Moreover, given that the Nature world is generated from a fine fireball in which the volume is smaller than an atom through a big-bang $[12,13]$, the basic component or fundamental particles of the matters should be much smaller than current known particles. Due to the limitation of current developed technologies, it is impossible to probe the substructure of the elementary particles or ultimate structure of matters by means of scientific apparatuses currently. Hence adopting suitable concepts from estimations, depended on current basic scientific knowledge, to illustrate the natural phenomena or doubts may be a reasonable means of approach [14, $15]$. 
Therefore in this work, through proposing a new concept from four simple hypotheses, namely the basic unit of particle or energy transporter, repulsion of particles of the same polarity, and the construction process of all particles and matters from the basic particles (basicles), the above mentioned scientific doubts can be readily explained. For instance, the reasons for the scattering of moving particle beams, thermodynamic laws, balance conversion of matter and energy, the component of dark energy/matter, as well as a minimum temperature of $\sim 3 \mathrm{~K}$ in the deep Universe and other scientific puzzles can also be clearly elaborated.

\section{Hypothesis}

\section{Hypotheses And Discussion}

$1^{\text {st } h y p o t h e s i s . ~ A l l ~ m a t t e r s ~ a n d ~ p a r t i c l e s ~ c o n s i s t ~ o f ~ t h e ~ s a m e ~ b a s i c ~ p a r t i c l e s ~(b a s i c l e s) . ~ A s ~ a n ~ e l e m e n t a r y ~}$ unit, a basicle has a polar property and cannot be further decomposed. Hence it represents the smallest unit or basic component of matters.

$2^{\text {nd }}$ hypothesis. Based on the polar property, basicles in different arrangements (during combination under exterior energy) form diverse types of bigger fine composite particles with diverse properties. The various similar or different fine composite particles further aggregate to even larger fine composite particles, which possess greater differences in properties and behaviours, in more diverse arrangements. Through these continuous aggregations or combination, the reported elementary particles (fermions and bosons) and big composite particles, such as well-known neutrinos, neutrinos, baryons, photons, phonons, electrons, protons, neutrons, nucleus, and so on [16 - 18], are generated (Fig. 1). As compared to a big particle (e.g., photon), most of the fine particles are likened to be small golf balls in contrast to the moon or a star in dimension. Thus they are invisible and undetectable by means of present technologies, but can possibly be found with the development of precise detection equipment in the future. Some fine particles of diverse forms are relatively stable whereas other unstable ones may be readily converted to new types of particles. From these dense/big and sparse/fine particles, different atoms, molecules, and various types of matters or antimatters (probably consisting of special atoms, in which the positions of proton and electron of common atoms are replaced by antiproton and positron, respectively) are well-known to be subsequently formed.

$3^{r d}$ hypothesis. Particles of the same type repel each other and the interaction of all particles and matters are dependent on the specific arrangements of other finer particles or basicles. Two basicles will not repel each other if they do not come into close contact. However, once larger particles are formed from two or more elementary basicles under an external force or energy, the as-generated particles of the same type will repel each other, as a result of the arrangement of basicles between and around them. Similarly, the attraction of particles of different polarity also occurs through the unique arrangements of finer particles or basicles. Due to the repulsion of similarly polarized particles, a larger dense particle consisting of compact uniform stacking of these finer particles in same types (including basicles) is usually unattainable unless under the influence of a quite high external force or energy (such as high speed bumping). Thus, similar to matters, all particles (excluding basicles) are expected to be porous when magnified under a 'super microscope'.

$4^{\text {th }}$ hypothesis. Matter is the transporter of energy while energy is the motion phenomena of matter. There is not any energy without matter.

\section{Discussion}

Based on the four hypotheses, we can explain numerous scientific doubts and laws of nature, such as the existence of different types or densities/sizes of particles, huge energy stored in atoms, balance of matter and energy conversion, particle beam scattering, thermal laws, and different time at the diverse atmosphere height around a dense planet or star. As the nondecomposed elementary basticles are the basic components of all matters and particles as well as the matter is the only transporter of energy, a balance between matter and energy can be achieved during their conversion since nothing is lost or added in the conversion. The basicles, represent the smallest units of energy transportation, are the basis of the conversion balance. Any radiation or energy is the phenomena of different motion or existence of particle(s) or matter(s), within which moving fine particles are contained. Thus this is the contributing factor to the $1^{\text {st }}$ Law of Thermodynamics - The total energy of an isolated system is constant, even though energy can be transferred from one to another type. In addition, as all particles such as atoms, are formed under a high external force or energy, they store huge energy inside. Some energy can be released when the particles decompose or combine to new particles, such as during the explosion of Hydrogen or atom-bomb, although the alteration of the relatively stable particles is usually required some extent of initial threshold energy. The repulsion of particles, such as photons, phonons, electrons, etc, of the same polarity usually leads to the scattering effect of the particle beam during moving forward. In addition, there are different interactions between particles of diverse properties, such as attraction between positive and negative particles. As the fine particles exist in huge quantity with different densities/sizes and diverse properties in the Nature world, the behavior of a particle is affected by other similar or diverse type particles or enclosed sparse or dense 


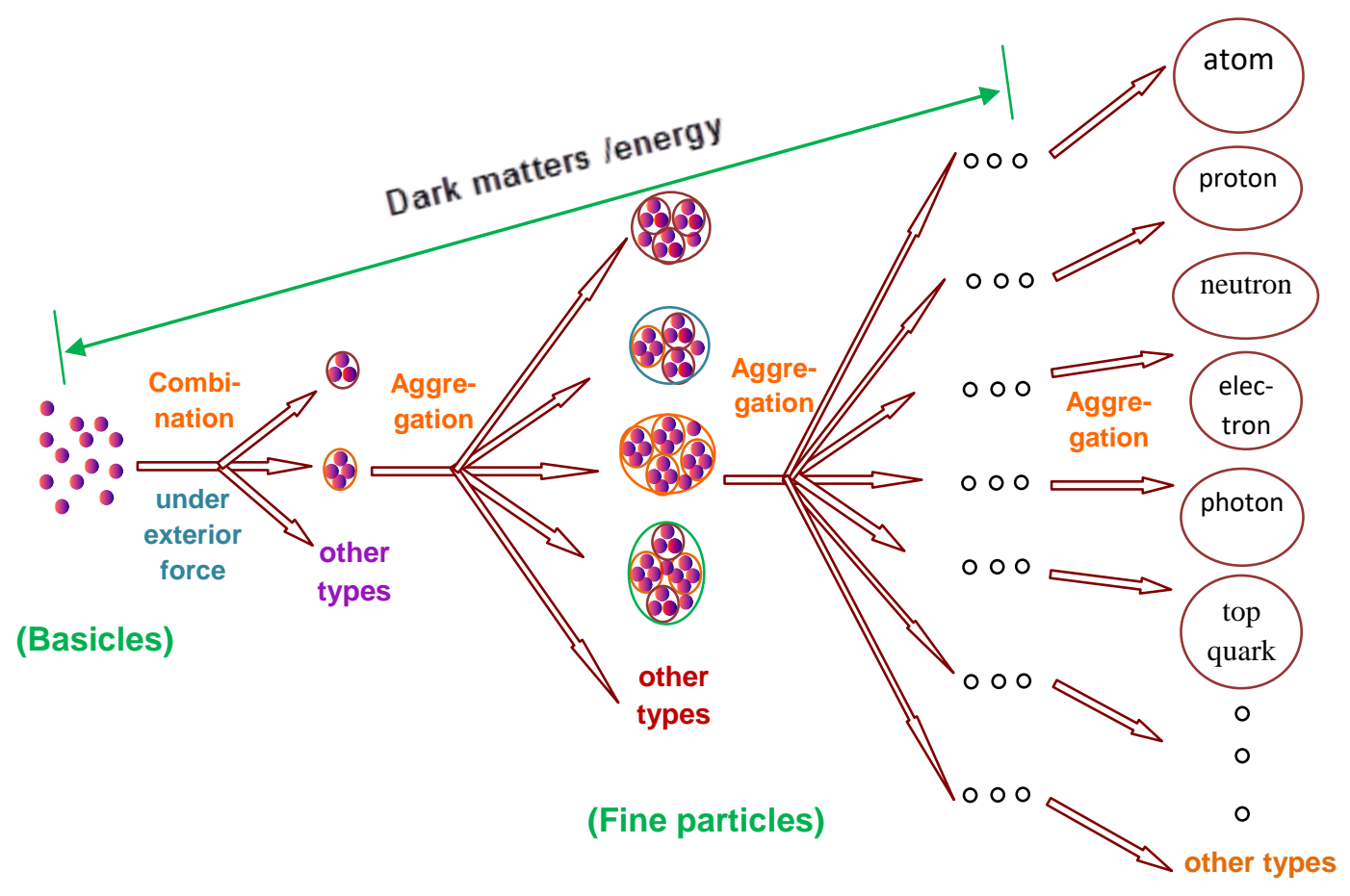

(Big particles)

Figure 1. Schematic illustration of all particle formation from the fundamental basicles through various combination or aggregation under external force or energy. The dark energy/matter is consisted of all invisible particles.

matters through the gravitational or other natural fields. This leads to different behaviors of specific particle in different environments. For example, most particles at atomic or sub-atomic scales (e.g., photons, electrons) behave as two phase properties of 'wave and particle', in which the position and motion direction of each particle cannot be simultaneously identified [19]. In addition, the repulsion of similar particles (e.g., photons, phonons) and elementary basicles (when in close contact) results in a tendency to usually occupy large volume where possible. This is likened to gas molecules moving from the bottle opening through the entire bottle space with a preference for larger entropy. This provides a possible explanation for the origin of the Second Law of Thermodynamics - In an isolated gas or particle system, the entropy approaches a maximum value [20, 21]. In considering the whole Nature world as a huge isolated system, the Second Law of Thermodynamics is taken into account here as well. Based on the four hypotheses, the particle distribution around a matter (Fig. 2a and b) as well as the dark energy or fine dark matter (Fig. 1) can also be explained. The distribution of particles around matters and in the Nature world can be readily predicted, as shown in figure 2a. The matters or dense/big particles, made up of various types of fine particles and basicles, can decompose into the sparse/fine particles and elementary basicles under different situations, such as bumping, heating, aging, degrading, decaying, etc. Hence there exists a lot of visible (e.g., photons) and, in particular, invisible particles of various kinds or densities/sizes surrounding all matters, such as stars, planets, and debris. As a result, vast types of particles occupy the whole Nature world or Universe. Hence the supposed 'vacuum' at the outer space of the Earth, where there exists negligible or no gas molecules, would also be made up of vast fine particles (such as radiation particles, photons, baryons, other finer particles, etc) instead of without any matter. Therefore, the Nature world and all matters are submerged in a sea of vast types of particles; there is dark energy or fine dark matter (Fig. 1) in the Nature world. Hence the absolute average temperature measured so far is usually higher than or at least $2.7 \mathrm{~K}$ in the deep spaces of the Universe $[22,23]$. The dark energy and fine dark matter also contain 'cosmic microwave background (CMB)' from the decaying radiation, suggesting the presence of photon particles as well. It is these vast invisible particles which constitute dark energy or fine dark matters, which total percentage is much higher than visible matters in Nature. Due to the effects of gravitational and magnetic fields of the dense matter, different types of visible (e.g., vapors) and invisible particles lie uniformly in a layer-like fashion around the dense or large matter (Fig. 2a), such as a planet or star. Bigger particles (e.g., $\mathrm{H}_{2} \mathrm{O}, \mathrm{O}_{2}, \mathrm{~N}_{2}, \mathrm{CO}_{2}$ molecules) and fine/denser particles occupy 

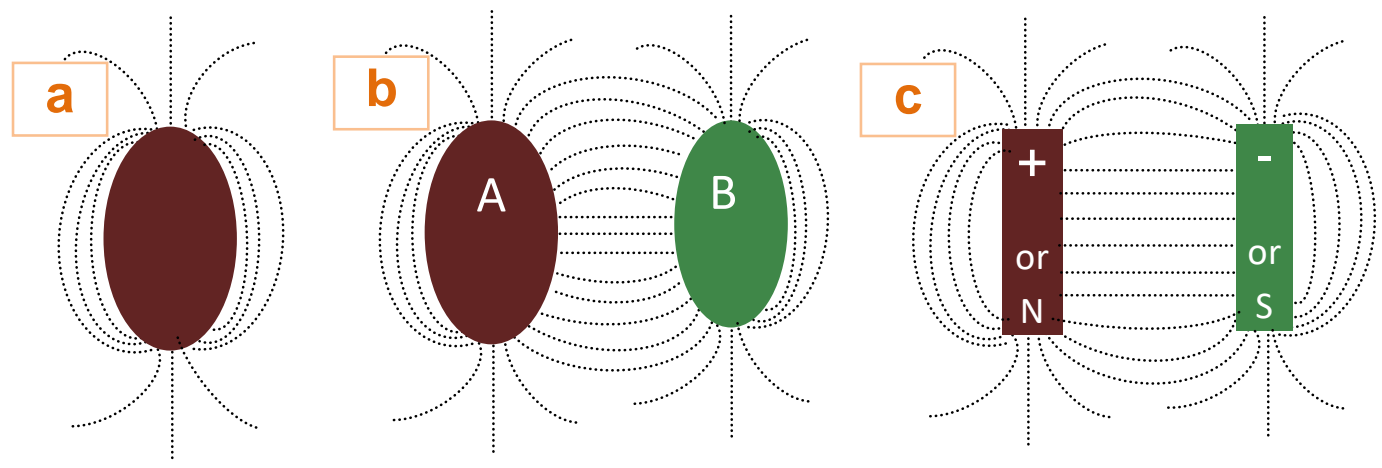

Figure 2. The arrangement of diverse fine particles (a) around a matter, and as the interaction media (b) in gravitational field between (A) and (B) matter as well as (c) in electrical or magnetic field, which are predicted from the four hypotheses.

in the inner layers, while only sparser/finer invisible particles reside at the outer layers. The distribution density of particles is reduced with increasing distance away from the matter (Fig. 2a). Within the same layer, the particle density and properties are usually similar. Hence their motion or decaying speeds as well as the attraction from other particles and the response to other particles are similar. Thus, the time within the same layer is the same, but differs slightly from the time at other layers. Time is well known to be measured by the internal motion or decaying speed of one matter at a specific location in the space. There are different times at different particle layers due to the diverse internal motion or decaying speeds of matter, which are determined by the different interactions among the matters and/or particles at the different layers. Particles (e.g., photons), in the presence of negligible gravitational effect, travel with greater ease along the same layer, compared to across different density particle layers around a matter. This may be the root cause of a bent space-time around a dense matter stated in Einstein's general theory of relativity.

Furthermore, the inter-media of the gravitational field, as well as electrical or magnetic field can be also explained based on the hypotheses. Due to the interaction between matters and/or particles, when two matters move close enough, different types of particles in the particle sea around the two matters arrange in an unique manner, depending on the properties and dimensions of the matters and particles, as shown in Fig. $2 \mathrm{~b}$. This arrangement process involves the transfer of interaction force from one matter to another through the huge quantity of invisible particles. This is the formation of gravity. Similar to gravitational force, the interaction media in magnetic and electrical fields are also attributed to the unique arrangements of specific invisible particles between and around the 'North' and 'South' magnetic poles or 'Positive' and 'Negative' electrodes (Fig. 2c). Therefore, all matter or field interactions occur through the different arrangements of invisible specific particles in the Nature world.

\section{Conclusion}

Through proposing the four hypotheses, the origins of some basic natural phenomena or Laws of Nature have been illustrated, such as the elementary component of particles and matters, particle beam scattering, balance of matter and energy conversion, huge energy stored in atoms, interaction media of Nature fields, components of dark matters or energy, Thermodynamic laws, different time at the diverse atmosphere height around a dense planet or star and so forth. These in turn can probably lead to further explorations of other scientific puzzles, such as the extinction and generation of the Universe.

\section{References}

[1] P.C.W. Davies, A new theory of the Universe, Nature, 255, 1975, 191-192.

[2] J.D. Barrow, The book of Nature worlds - Exploring the limit of the Cosmos (New York: W. W. Norton \& Company, 2011).

[3] N.S. Agarwal, Experimental proof of mass in photon, J. Mod. Phys. 6, 2015, 627-633.

[4] S. Weinberg, The discovery of subatomic particles 2nd Edition (Cambridge: Cambridge University Press, 2003).

[5] N. Turok, Particle physics: Particles and the Universe, Nature 322, 1986, 111-112.

[6] B. Carithers and P. Grannis, Discovery of the Top Quark, SLAC Beam Line 25, 1995, 4-16.

[7] E. Akkermans and G. MontambauxJuly, Mesoscopic Physics of Electrons and Photons (Cambridge: Cambridge University Press, 2006).

[8] R. McWeeny, Atoms, molecules, matter - the stuff of Chemistry (Pisa: University Pisa, http://www.learndev.org /ScienceWorkBooks.html, 2007)

[9] A.R. Wallace, Man's place in the Nature world 4th edn (London: Chapman and Hall, 1912).

[10] D. Lincoln, The Large Hadron Collider: The extraordinary story of the Higgs Boson and other stuff that will blow your mind. (Baltimore: Johns Hopkins University Press, 2014).

[11] J.D. Lykken, Beyond the Standard Model (CERN: CERN Yellow Report pp 101-109, 2010). 
[12] L.M. Krauss and R.J. Scherrer, The return of a static universe and the end of cosmology, Gen. Relativ. Gravit. 39, 2007, 1545-1550.

[13] P.J.E. Peebles, D.N. Schramm, E.L. Turner, and R.G. Kron, The case for the relativistic hot big bang cosmology, Nature 352, 1991, 769-779.

[14] J.D. Barrow and M.P. Dabrowski, Oscillating universes. Mon. Not. R. Astron. Soc. 275, 1995, 850-862.

[15] J. Gribbin, In search of the multiverse (Allen Lane, New York: The Penguin Books Group, 2009).

[16] H. Dingle, Science and the unobservable, Nature 141, 1938, 21-28.

[17] I. Kant, Universal natural history and theory of the Heavens (Ann Arbor: University of Michigan Press, 1969).

[18] A.W. Thomas and J.D. Vergados, Solar neutrinos as background in dark matter searches involving electron detection, J. Phys. G: Nucl. Part. Phys. 43, 2016, 07LT011-07LT019.

[19] L. Boltzmann, On certain questions of the theory of gases, Nature 51, 1895, 413-415.

[20] S.A. Bludman, Thermodynamics and the end of a closed Universe, Nature 308, 1984, 319-322.

[21] E. Gunzig, J. Géhéniau, and I. Prigogine, Entropy and cosmology, Nature 330, 1987, 621-624.

[22] J.V. Narlikar and N.C. Wickramasinghe, Microwave background in a steady state Universe, Nature 216, 1967, 43-44.

[23] A.C.S. Readhead, S.T. Myers, T.J. Pearson, et al. Polarization observations with the cosmic background imager. Science 306, 2004, 836-844. 